\begin{abstract}
Audiences, who cannot investigate the credibility of most news stories for themselves, rely on non-content heuristic cues to form credibility judgments. For most mediums, these heuristics were stable over time. Emerging formats of journalism, however, require audiences to learn to interpret what new heuristics credibility cues mean about the credibility of the story. In an experiment, participants $(\mathrm{N}=254)$ were given instructions about how to interpret the credibility cues in three formats as they read a politicized news story, which were compared to a control condition that did not have any instructions. The results show the timing and source increase the effectiveness of the instructions. Partisanship, though, remains the driving force of credibility perceptions.
\end{abstract}

Keywords: partisanship, credibility, heuristic cues, news, media literacy. 


\section{THE EFFECTIVENESS OF CREDIBILITY INDICATOR INTERVENTIONS IN A}

\section{PARTISAN CONTEXT}

The newly launched Colorado Sun website embedded in news stories a box of credibility indicators. In this box, readers can click to learn more about whether the story is original, on-theground reporting, whether the reporters are subject specialists, and what sources are cited in the article ("About Us," 2019). The funding organization for the Sun, Civil, cited the difficulties audiences have with media literacy as a major reason for the credibility indicator box (Himmel, 2018). As public discourse in the wake of the 2016 presidential election turned to the role of hoax news stories that spread across social media sites like Facebook, audiences said they were having difficulty telling the difference between credible news and hoax news. A late-2016 Pew survey found 64 percent of American adults said "completely made up" news was causing them a great deal of confusion about current events and facts (Barthel, Mitchell \& Holcomb, 2016). Further, about $23 \%$ of those same adults said they had shared a political news story that was fabricated, whether they knew at the time it was made-up or learned later.

To bring audiences up to speed, several news organizations responded with "How to" articles on spotting "fake" news: The Globe and Mail offered a list of tips such as paying attention to news trusted news brands and URLs (Annett, 2017). The Associated Press gave audiences of a list of five steps to take that included clicking the link then reading the story, and paying attention to grammar and emoticons (Ortutary, 2016). Facebook, for its part, listed 10 heuristics to which to look when determining credibility (Facebook, 2017). Similarly, the News Literacy Project distributed tips for as part as a news literacy program for adoption in classrooms across the country, teaching students what to be wary of when reading news online (Foley, 2017). 
The appeal of heuristics as a checklist to aid in media literacy worked when the news media environment was stable, but the continued evolution of online news design makes the usefulness of any one list ephemeral. Marsh and Yang (2017) said teaching audiences to focus on source evaluation as a form of media literacy will not work because "typical cues for credibility have been hijacked" to blur the lines between news that is malicious and news that aims to tell the truth. At the same time, the sheer magnitude of information available from innumerable news brands means newsreaders must rely on heuristics and social cues to assess information from unfamiliar news organizations (Lazer, Baum, Grinberg, Friedland, Joseph, Hobbs \& Mattsson, 2017). Of particular concern is media literacy skills to surrounding news about politics. Research shows political news can trigger a defensive response in audiences that makes them resistant to updating their beliefs (Festinger, 1957; Lowin 1967). That reaction has recently been applied to the tendency of audiences to consider news that challenges their worldview as "fake."

Together, these concerns expose three gaps in the literature of vital importance for today's news literacy and participation in democracy. First, how can news organizations teach audiences to understand and interpret the credibility heuristics available to them? Second, how does teaching these heuristics to audiences affect their perception of news credibility of political news? Finally, how does the partisanship of news audiences aid or impede their ability to learn credibility heuristic cues on political news? There's little research on how adults learn best to tell the credibility of the news in the face of changing technology and expanding availability of news. One exception is a report from the Center for Media Engagement, which found that adding a box next to news stories which explains the reporting process can increase reader trust in that story (Chen, Curry \& Whipple, 2019). Additional understanding about learning credibility heuristics comes from child development literature that observes children as they develop a sense 
of interpersonal credibility. But, adults must continue to develop a system of determining information credibility as the ways they access news changes.

The purpose of this study is to test several easy-to-implement cognitive interventions to teach audiences how to understand and interpret credibility heuristic cues, and how ideology plays a part in learning these cues about partisan news. In a survey-embedded experiment, it looks at three formats of interventions in particular: A pop-up before the news story provided by the news site itself, a sidebar next to the story with the same information as the pop-up, and a sidebar next to the story with tips provided by other users. These formats are compared to a control condition, in which participants were provided no additional information about how to interpret the credibility heuristics.

\section{Literature Review}

\section{News credibility}

How audiences perceive the credibility of the news they read is the subject of more than six decades of research that began with Hovland and Weiss (1951) because it is vital to understanding how citizens make decisions in a self-governing society. While the practices, routines and conventions of journalists are designed to cultivate credibility (Coddington, 2014; Tuchman, 1978), the decision about what to believe is ultimately in the eyes of the audience (Gunther, 1992). While logical, analytical and careful consideration of the argument is the mostprized type of message processing when making credibility judgments, people tend to be cognitive misers to make more efficient decisions (Fiske \& Taylor, 1991). This means that audiences will process no more cues than they determine is needed to make a relatively risk-free decision about the credibility of news (Sundar, Knoblach-Westerwick \& Hastall, 2007). Pjesivac, Geidner and Cameron (2018) found modern Internet news readers primarily use the peripheral 
processing route, paying attention to heuristics like source expertise rather than the content of news messages. Yet, it seems, when audiences encounter obviously not credible information, they may more systematically process the news by spending more time with it and demonstrating better recall of it than credible information (Geidner \& Cameron, 2017).

Heuristic credibility systems change each time a different news delivery system emerges Because when audiences first find news on an unfamiliar medium, the modality, design of the interaction and the story structure are different from past experiences with media (Kiousis, 2001; Lee, Park, Lee \& Cameron, 2010; Sundar, 2008), audiences must adjust to the heuristic cues provided by these mediums if they are to reap the benefits of the medium such as convenience and quick delivery to audiences. Transitioning to a new medium causes confusion and disorientation (McDonald \& Stevenson, 1996; Bucy, 2003), and audiences are less able to identify cues that were once important to determining credibility such as message source (Vraga, Edgerly \& Wang, 2011). This has been a particular concern as newspapers move online and make changes in the visual design of news (Li, 2002; Lowrey, 1999).

While the connections between systems of heuristic credibility cues and the perception of credibility seem obvious, much of the literature focuses on a checklist approach to researching how cues affect the perception of credibility. Studies isolate heuristics such news brand names (Metzger, 2007; Sundar \& Nass, 2001), source credibility (Pjesivac, Geidner \& Cameron, 2018), platform publisher (Bhandari, 2018), number of social recommendations (Xu, 2013), or website features (Flanagin \& Metzger, 2007) to find the effects of cues on the perception of credibility. But, little research looks at how knowledge about the interpretation of these heuristics is acquired, and the role that partisanship - which is known to create knowledge gaps in coordination with media use (Hindman, 2009) - might play in the ability of the audience to learn 
and interpret these cues. However, the suggestion that audiences can learn news credibility heuristics comes from two areas of the literature.

\section{Learning to judge credibility}

First, theory can draw on early childhood literature to offer suggestions that credibility cues can be learned. Flanagin, Metzger and Hartsell (2010) found in a survey of school-aged children that the more the students were exposed to Internet information the more they were able to identify the importance of using analytical cues to weed the false information from the true information. The students, though, reported they more often relied on heuristics like a "gut reaction" or "feelings." Birch, Akmal \& Frampton (2010) demonstrated that 2- and 3-year-olds could learn credibility cues. When only one adult tried to teach them, with or without non-verbal credibility cues, the children learned equally. But, when they saw more than one adult teaching, some with credibility cues and some without, the children showed preference and learned more from the adults who demonstrated credibility cues. More sophisticated cue use in interpersonal communication, like a motivation to be biased toward a belief, may not develop until middle school (Mills, 2013).

Second, survey evidence suggests that audiences adjust to these systems over time. The more people use a news medium, the more they consider it credible (Kiousis, 2001). Crosssectional data suggests that as news technology becomes more prevalent and more familiar, the perception of credibility of news on that technology increases (Johnson, 1969; Johnson \& Kaye, 2004; Mackay \& Lowrey, 2011). Audiences, however, are able to develop platform-specific heuristic cue sets (Chung, Nam \& Stefanone, 2012). For example, modern audiences are judging political news on social media by different criteria than they judge political news found on blogs, news websites, or broadcast television (Johnson \& Kaye, 2014) and continue to find blogs less 
credible than traditional news (Bhandari, 2018). The evidence that audiences can learn cues to credibility and that as they grow familiar with the cues that the perception of credibility increases suggest a positive correlation between the interpretation of credibility cues and the perception of credibility.

\section{Partisanship and credibility judgments}

Though the literature suggests that audiences should be to learn credibility cues, partisanship can also influence news media literacy outcomes. Vraga and Tully (2015) showed partisan audiences a clip of a television talk show about immigration reform in which the host took either a liberal, conservative or neutral position. With half of the audience, they intervened with a PSA promoting the importance of media literacy before the clip ran. They found that conservatives who saw the PSA before the conservative-host clip rated the host and the program as more biased. That perception of bias though did not alter their perception of credibility. Liberals who saw the PSA questioned their own motives and so saw the conservative host as neutral. Where news media literacy is taught, Democrats are more likely to increase their belief in the value of news media literacy (Tully \& Vraga, 2017). Similarly, Chen, Curry and Whipple (2019), were able to improve the audience's perception of credibility of a news story by placing a "Explain the Process" box on both mock and real news stories. Yet, the box did not reduce the audience's perception that the news organization "had an agenda." Further, the authors found a similar addition to news stories designed to reduce the perception of partisan bias in the news article was not effective enough for the authors to recommend adoption by newspapers (2019).

Partisanship may present a barrier to learning credibility heuristics and updating beliefs because of cognitive processes that create a tendency to interpret the world in a way that favors maintaining the status quo. Partisan audiences have the tendency to believe information when it 
confirms their previously existing beliefs (Festinger, 1957; Lowin, 1967). But beyond swaying opinions, the partisan heuristic about a politicized issue can change the belief about factual information. This pattern of "selective learning" (Jerit \& Barabas, 2012) shows what partisans tell pollsters about objective facts like crime rates are filtered through their political worldview. Jerit and Barabas $(2009,2012)$ found that media coverage of a politicized issue exacerbated the knowledge gap along partisan lines. The more news media report on an issue, the more likely people report that they know more facts that help their party or hurt the opposing party in comparison to facts that would be detrimental to their party (Jerit \& Barabas, 2012). Partisans are able to dismiss attitude-discrepant information sources that have a lower credibility more easily than attitude-discrepant information from sources with high credibility.

When it comes to politicized news issues, credibility also plays a part in the information choices of partisans. Westerwick, Kleinman and Knobloch-Westerwick (2013) in selective exposure experiments found when personal relevance was low, users spent more time with highcredibility sources. But, when personal relevance was high, they favored attitude-discrepant messages from low credibility sources compared to high credibility sources. This suggests the audience will take extra steps to avoid attitude inconsistent information when it is an important issue, even taking the risk of being misinformed by a low-credibility source.

H1: Partisans will learn fewer credibility cues than non-partisans.

H2: Partisans will rate a story about a politicized news story as less credible than nonpartisans.

\section{Timing and credibility}

The tendency of audiences to choose news and to believe ideas that agree with their political view is well established. Explanations about what can increase the audience's 
willingness to read, consider or believe an opposing viewpoint are less clear. Two interventions that seem to increase the perception of credibility while simultaneously suppressing the effect of partisan cues emerge from the literature: The timing of the information given to the audience and positive social recommendations. While the role of these factors is not directly addressed in news credibility literature, the theories and circumstances of these effects are similar enough that they may provide guidance to how audiences process information meant to teach them how to interpret credibility heuristics.

First, evidence suggests that the timing of credibility cues will matter to a skeptical audience. Sternthal, Dholakia and Leavitt (1978) first found that when evaluating the persuasive ability of consumer messages that the message source's degree of credibility only had an effect when it was provided before the message. Where an audience member was negatively predisposed to a message and the source of the message was revealed after the consumer saw the message, whether the source was high or low credibility made no difference on the persuasiveness of the message. Similarly, Vraga and Tully (2015) found an effect of a media literacy PSA on partisan audiences when it was placed before political talk show television clip.

Tormala, Briñol and Petty (2007) expanded these findings by thoroughly examining the role of timing of the identification of a message source in persuasive messages. In an experiment, participants were told the source of a message about at a chemical in soap detergent was either the government (high credibility) or a manufacturer of detergent (low credibility). The source of the message was revealed to the participants either before the message or after the message. When the source was revealed before the message, the credibility manipulation influenced the valence of thoughts so that high credibility sources invoked more favorable thoughts. When the 
source of the message was revealed after the message, the credibility of the source had no effects on the audience's thoughts about the message.

These studies suggest that when a high-credibility source of a message is revealed to the audience, it can change how the audience processes the message so that members are more likely to view the message favorably. Tormala, Briñol and Petty (2007) suggest this effect is observed because the audience is predisposed to believe the message and hence spends less cognitive effort on examining the content of the message. This evidence suggests the timing of the when source credibility information matters to the audience's perception of credibility. This study posits that similarly the timing of when information about how to interpret the credibility cues about a news story will have an effect on the audience's perception as well.

H3: Audiences will learn more heuristic cues when instructions are provided before a politicized news story than when instructions are provided simultaneously to a politicized news story.

\section{Social recommendations and credibility}

The second factor, social recommendations, is an emerging line of literature that reflects the gaining popularity of "crowdsourced" heuristics made possible by interactive internet technology. Social recommendations - the suggestions of non-elites through individual expressions or aggregated opinions - showed to be powerful heuristic cues in several studies. Metzger, Flanagin and Medders (2010) found in focus groups that audiences relied on the recommendations of others to make credibility decisions about monetary transaction websites, such as Amazon or Paypal.

Applied to the context of online credibility judgments, when choosing between sources, people are likely to believe that a source whose name they recognize is more credible compared to unfamiliar sources. People appear to reason that name 
recognition is earned by positive interactions overtime that are spread through social networks. (p. 426)

Further, they found that when others recommended or endorsed a website, participants put more faith in that heuristic than their own first-hand experience with a website. "Regardless of their previous opinion on a product, some participants noted that they often used comments and testimonials to direct their purchase decisions" (p. 428). In an experiment, Xu (2013) found increasing the number of social recommendations on a social networking platform increases both the likelihood of clicking on a news story and the perceived credibility of the news. This study found that as the number of more "upvotes" a story received on Reddit increased, the audience's perception of credibility of the story increased.

Because these studies find that social recommendation is a powerful heuristic in determining online credibility, this study hypothesizes that instructions on how to interpret news credibility heuristics provided through social recommendations will positively affect the audience evaluations of the message.

H4: Social instructions about the interpretation of heuristic credibility cues will increase the number of cues the audience can correctly identify compared instructions from the website.

\section{Method}

These hypotheses were tested using a mock mobile news application. This context was used because it is an emerging medium for news that continuously changes. These circumstances mean that the absence of unfamiliar credibility heuristics would not communicate a negative credibility and that the audience would not have any pre-existing knowledge about how to interpret interpretation credibility heuristics. The stimulus, therefore, manipulated the source and timing of the instructions about how to interpret the credibility heuristics in an environment 
similar to what happens when news technology creates major shifts in the availability of news credibility cues.

\section{Participants}

Adults residing in United States $(\mathrm{N}=254)$ were recruited by Survey Sampling International and paid incentives by the service for participating. The participants were mostly white $(81 \% ; 8 \%$ Asian or Pacific Islander; 7\% African American or Black; 7\% Hispanic). A slight majority of participants $(55 \%)$ were men. The average participant aged 47 . The participants identified their party affiliation as $31 \%$ Democratic; $45 \%$ percent Independent; and 24\% Republican. This study received approval through the researcher's Institutional Review Board.

\section{Procedure}

Participants contacted by SSI were told that they were helping to evaluate a mobile news application before it was available to the public. This application, participants were told, employed an algorithm to assess the credibility of news aggregated from multiple news sources and assign it credibility "grades." After answering a few pre-test questions about media use, opinions about current news topics, and attitudes about technology use in news delivery, the survey software assigned participants to one of four conditions. All participants saw the same news story with the same credibility heuristics. Depending on condition, the instructions about how to interpret the credibility heuristics in a column to the right of the story or on a screen before the news story. Those in the control condition were not given any instructions on interpreting the cues. Participants were able to view the experimental stimulus on internetconnected devices, including smartphones, tablets and laptop or desktop computers, simulating many mobile applications that are available for different sized screens. Audiences were able to zoom in to see any detail if they wanted. 
After reading the news story, participants answered questions about how they interpreted the credibility heuristics and perceived the credibility of the news story. After several demographic and sociographic questions, participants were debriefed about the use of deception in this experiment.

\section{Stimulus}

The news story used here was written by a former journalist to be an unbiased news story about a politicized issue. The short news story concerned a new technology tool available to police departments to fight crime. While the tool was expensive, a lawmaker with ties to the manufacturer of the tool was proposing a bill that would force departments to buy it. It followed journalistic conventions and included information from both sides of the issue. The decision to use a mock news article was made to increase internal validity on two levels: First, this ensures that the audience had no prior experience with the issue that could bias their perceptions. Second, the news app was designed to have a slightly futuristic look as to provide the audience with an unfamiliar heuristic environment and replicate conditions where participants would need to learn to judge credibility.

The topic of crime was chosen because crime and policing was politicized during the 2016 presidential campaigns. A Pew Research Center survey found that perceptions of crime rates differed by support of candidate (Gramlich, 2016). While 37\% of those who supported Hillary Clinton's bid for the presidency said that crime in the United States worsened since 2008, $78 \%$ of those who supported Donald Trump felt the same. The same report noted that FBI's statistics found crime decreased about 20 percent during the same time period. Therefore, it was an appropriately partisan issue when this experiment was fielded in March 2017. 
The news story made visible three specific design elements that were indicators of credibility. This means to interpret the credibility heuristics of the news story, the audience had to learn their meanings. However, these cues are ones not currently used in other news environments. For example, the story gave a number of "points." The points had no context on the layout of the page, but the instructions explained that the points indicated how reliable the news story was on a scale of 1 to 200 .

The experiment provided instructions on their interpretations in three of the four conditions. The fourth condition, the control condition $(N=65)$, provided the audience no way to assess or interpret these three design elements. While this condition did not provide any information on how to interpret the cues, the cues were visible. This decision was made to ensure it was the participants' ability to learn the meaning of the cues that made a difference in their perception of credibility rather than the presence of this design element. To operationalize the factors that the literature suggests would improve the ability to learn credibility heuristics in an externally valid way, audiences viewed three conditions in a controlled survey-embedded experiment. To examine the role of timing, audiences were shown the exact same wording for the instructions in two conditions. In the "site tips" condition $(N=64)$, instructions for interpreting the credibility heuristics were provided in a sidebar that was shown to the audience at the same time as the news story.

In the "pop-up" condition ( $N=66)$, the instructions were provided to the audience on a screen where they could only read the instructions. Next, they saw the same story as those in the control condition. An introduction sentence in these conditions suggested that the source of the instructions was the site itself through pronoun usage. 
To examine the role of social recommendations, the "user tips" condition $(N=59)$ featured tips about how to interpret the credibility heuristics were displayed in a sidebar titled "What Readers Are Saying." Short, tweet-length statements appeared in this column. Each of the tips was labeled with a username. Additionally, the pronoun usage in these tips was altered to indicate that the source of the tips was individual readers.

\section{Variable construction}

Credibility. The perception of credibility was measured through six questions measured on scales from 1 to 5, where larger numbers indicate higher perceived credibility. These items were adapted from Meyer (1988), which has been used to measure audience perceptions of news message credibility in emerging online news contexts (Kiousis, 2001). Participants rated the news story on the degree they thought the story was: interesting, fair, accurate, believable, and credible. They also rated the degree to which they thought the story showed bias in favor of lawmakers and in favor of law enforcement. The bias questions were reverse coded and averaged to create one bias item. The Cronbach's Alpha for the six items was .734, indicating a reliable index. Then the six items were averaged to create the credibility index $(M=3.55, S D=0.59)$.

Cues correctly identified. After the participants read the news story and instructions, all participants including those in the control condition were asked to recall the interpretation of three credibility heuristics. Participants were given one point for a correctly identified heuristic and zero points wrong answers and do not know answers $(M=1.09, S D=1.06)$.

Participant partisanship. Participants were asked to rate their political party partisanship on a scale from 1 to 7 , where 1 was strong Democrat and 7 was strong Republican $(M=3.82, S D=1.85)$. Participants who identified as strong, moderate, or leaning Democratic were grouped as Democrats $(N=78)$. Those who identified as strong, moderate or leaning Republican 
were grouped as Republicans $(N=59)$. Those who identified as independent were so categorized $(N=113)$.

\section{Results}

Manipulation checks. To see check how well the manipulation of the source of the instructions for the credibility heuristics worked, participants were asked to recall the source. In the three treatment conditions, the correct answer was the most common answer (site tips $N=16$; user tips $N=10$; pop-up $N=25$ ). A Pearson's chi-Square shows an association between the two categories, $\mathrm{X}^{2}(9,105)=23.37, p=.005$.

To check to see if the manipulation of the format of credibility instructions changed the degree of scrutiny participants gave the content of the news story, participants were asked two recall questions about the content. Participants were given one point for a correct answer and zero points for an incorrect answer or a "don't know" answer. A one-way ANOVA of those content recall questions by condition found no significant difference, $F(3,253)=1.317, p=.269$.

To check that participants interpreted the credibility cues correctly, a Pearson correlation between the number of cues learned and the perceived credibility was used. The results indicate a significant positive correlation of $r=0.182, p=.004$. This suggests that as the audience is able to identify more credibility heuristics correctly, it perceives the credibility of the news story as higher.

Hypothesis testing. To examine the set of hypotheses predicting that political partisanship and condition would affect the number of cues learned and the perception of credibility, an ANOVA was used. Results suggest that condition had a main effect on the number of cues learned, $F(3,249)=10.98, p<.001$. Pairwise comparisons with Bonferroni correction shows that those in the pop-up condition learned more credibility cues than those in the control 
condition $(M D=.97, S E=.19), p<.001$; and more than those in the site tips condition $(M D=.57$, $S E=.18), p=.012$. Further, those in the user tips condition learned more than those in the control condition $(M D=.83, S E=.2), p<.001$. There was no main effect nor an interaction of participant partisanship on the number of cues learned. See Figure 1. H1, which suggested that partisanship would inhibit learning credibility cues, was not supported. These results support H3, which suggested that an intervention before a news story would increase learning compared to an intervention simultaneously to the news story. The results also provide evidence to support $\mathrm{H} 4$, which suggested that social recommendations about learning credibility cues would increase learning compared to the control condition.

To address the final hypothesis, an ANOVA was used to test the effect of participant partisanship and condition on the perceived credibility of the news story. Participant partisanship was a significant predictor of the perceived credibility, $F(2,249)=4.17, p=.017$. Multiple comparison tests show that Democrats rated the news story as more credible than independents ( $M D=.30, S E=.11), p=.016$. Condition had no effect on the perception of credibility. There was no interaction. See Figure 2. These results provide support for $\mathrm{H} 2$, which suggested that participant partisanship would affect the perception of a neutral news story about a politicized issue. Here, partisan Democrats perceived the news story to be more credible than non-partisan independents.

\section{Conclusion}

This study used simple interventions in the form of instructions to teach participants the meaning of heuristic credibility cues, mimicking the conditions that audiences would face when they encounter news in an emerging journalism format. In a controlled experiment, these manipulations tested an unusual set of circumstances - one where the audience had no familiarity 
with the credibility heuristics but that unfamiliarity did not communicate a lack of credibility. In this context, the results presented here were able to complement the survey literature that finds that overtime audiences learn to adjust to new credibility heuristics to learn to distinguish news with high credibility from low credibility. Additionally, because the literature suggests that both learning and the perception of credibility are influenced by partisanship, audiences were given a story written with a balanced viewpoint about a politicized news topic to test the role of partisanship in the perception of credibility in absence of the usual markers of credibility.

The results of this study have interesting implications for theory and for newsrooms that are looking for ways to increase the audience's ability to determine the veracity of political news. These results separated the influence of pre-existing knowledge about credibility heuristics (or actual increases in the credibility of news on a new format) from the effect on perception of credibility. The increase in identified credibility cues cannot be attributed to more familiarity with the news or more familiarity with politics.

These results also suggest that two formats for instructions work best to teach audiences how to interpret credibility heuristic cues. First, audiences responded best to instructions that were given to the audience before the politicized news. Second, audiences learn the meaning of more credibility heuristics when the source of the tips is other users. These findings comport with prior research that audiences respond well to social recommendations and that timing matters when processing source credibility information. Further, while partisanship is known to interfere with learning facts, these results showed no evidence that partisanship would prevent differing levels of knowledge about the meaning of credibility cues. With these results, news literacy advocates can take care to provide explicit instructions on how to interpret news credibility heuristics through either a pop-up message before the politicical news or through 
crowd-sourced tips. Further, research could investigate if user-generated tips provided through a message presented before the audience reads the news can even further increase the ability of the audience to learn credibility heuristics. All of these formats for instructions were tested here in a controlled setting manufactured by the research. Developing and testing these formats on everyday audiences will be key to knowing how effective these types of interventions will be on mass audiences.

While differing formats for instructions have an effect on how many cues the audience learns, the format did not have an observable effect on the perceived credibility of the news story. Instead, though this story was written from a neutral viewpoint, it was the partisanship of the audience that influenced the perceived credibility of the news story. This finding aligns with what many advocates of media literacy and critics of the "cheerleader" partisans fear: differing perceptions of credibility based not on cues aimed at increasing credibility but rather pure partisanship.

The shifting main effects of instruction format and partisanship on the number of cues learned and the perception of credibility challenge the checklist approach to credibility research. Had this experiment ended at measuring the correlation between number of credibility cues learned and perception of credibility, it would have missed a major influence on the perceived credibility. By considering the partisanship of the audience as a factor influencing the perceived credibility, evidence suggests a sort of paradox where partisans are able to learn the credibility cues on par with non-partisans through the differing conditions. However, those same conditions do not have an equal effect on the perception of credibility. Instead, partisan mindset takes over and audiences are influenced by the political context of a story. Future research should 
investigate other politicized news topics and look at mechanisms that might explain how partisanship can be taken out of the equation for partisan audiences.

While these findings offer key insights, like all experiments, this study has its limitations. First, for ecological validity the design and wording of the user-generated tips were not the same as the site tips nor the pop-up tips. For this reason, the user-tips cannot be directly compared to either though effort was made to explain the same cues in the same way. Second, while it makes sense that no participant in the control condition correctly answered all three questions about the meaning of the credibility cues - they were given no information to be able to learn what the heuristics on the page meant, after all - this makes statistical comparisons between those who did answer all three correctly in the treatment conditions and the control condition impossible. Finally, the manipulation check to the source of the tips shows about half of the participants could not correctly recall who was providing the instructions about how to interpret the credibility heuristics. While a Chi-square shows the participants did pay some attention to the source, and while this attention likely replicates the attention audiences pay during the normal course of reading, the full effect of the credibility heuristic instructions may not be observed in this study. However, the results of this manipulation check also may be similar to real-world news attention. Mahone (2014) found similar numbers of participants were unable to remember the source of a news story in an experiment.

Nevertheless, this study has three key findings for mass communication scholars and media literacy advocates: Increasing the number of learned credibility heuristics in emerging news platforms increases the perceived credibility of the news when those heuristics indicate high credibility. Crowdsourced information about the meaning of credibility heuristics increase the number of credibility heuristics audiences learn, while information before a politicized news 
story increases the number of cues learned compared to when the information is displayed simultaneously to the news story. And, partisanship plays a dominating role in determining the perceived credibility of politicized news stories, regardless the format that credibility heuristic information is given to the audience. 


\section{Works Cited}

“About Us.” Colorado Sun. https://coloradosun.com/about-us/.

Anette, E. (2017). "What is fake news and how can you spot it?" The Globe and Mail. Retrieved from: https://www theglobeandmail.com/community/digital-lab/fake-news-quiz-how-tospot/article33821986/.

Barabas, J., \& Jerit, J. (2009). Estimating the causal effects of media coverage on policy-specific knowledge. American Journal of Political Science, 53(1), 73-89.

Barthel, M., Mitchell, A. \& Holcomb, J. (2016). "Many believe fake news is sowing confusion." Pew Research Center. Retrieved from http://assets.pewresearch.org/wpcontent/uploads/sites/13/2016/12/14154753/PJ_2016.12.15_fake-news_FINAL.pdf.

Bhandari, M. (2018). Social media cues and news site name: What do they mean for online news perception?. Newspaper Research Journal, 39(2), 169-179.

Birch, S. A., Akmal, N., \& Frampton, K. L. (2010). Two-year-olds are vigilant of others' nonverbal cues to credibility. Developmental science, 13(2), 363-369. DOI: 10.1111/j.14677687.2009.00906.x

boyd, d. (2018). You think you want media literacy. Do you? Medium. Retrieved from https://points.datasociety.net/you-think-you-want-media-literacy-do-you-7cad6af18ec2.

Bucy, E. P. (2003). Media credibility reconsidered: Synergy effects between on-air and online news. Journalism \& Mass Communication Quarterly, 80(2), 247-264. doi:

\subsection{7/107769900308000202.}

Chen, G. M., Curry, A., \& Whipple, K. (2019). Building trust: What works for news organizations. Center for Media Engagement. https://mediaengagement. org/research/building-trust 
Chung, C. J., Nam, Y., \& Stefanone, M. A. (2012). Exploring online news credibility: The relative influence of traditional and technological factors. Journal of Computer-Mediated Communication, 17(2), 171-186. DOI: 10.1111/j.1083-6101.2011.01565.x

Coddington, M. (2014). Defending judgment and context in 'original reporting': Journalists' construction of newswork in a networked age. Journalism, 15(6), 678-695.

Facebook. (2017). “Tips to spot false news.” Facebook. Retrieved from https://www.facebook.com/help/188118808357379.

Festinger, L. (1957). A theory of cognitive dissonance. Stanford, Calif; Stanford University Press.

Fiske, S. T., \& Taylor, S. E. (1991). Social cognition, 2nd. NY: McGraw-Hill, 16-15.

Flanagin, A. J., \& Metzger, M. J. (2007). The role of site features, user attributes, and information verification behaviors on the perceived credibility of web-based information. New Media \& Society, 9(2), 319-342. https://doiorg.ezproxy.library.wisc.edu/10.1177/1461444807075015

Flanagin, A. J., Metzger, M. J., \& Hartsell, E. (2010). Kids and credibility: An empirical examination of youth, digital media use, and information credibility. MIT Press.

Foley, R. (2017). "Efforts grow to help students evaluate what they see online." Associated Press. Retrieved from https://apnews.com/64b5ce49f58940eda86608f3eac79158.

Geidner, N., \& Cameron, J. (2017). Readers perceive deceptive graphics as less credible. Newspaper Research Journal, 38(4), 473-483.

Gunther, A. C. (1992). Biased press or biased public? Attitudes toward media coverage of social groups. Public Opinion Quarterly, 56(2), 147-167. 
Himmel, J. "Media literacy starts with defining credibility." Civil. Medium. https://blog.joincivil.com/tagged/credibility-indicators

Hindman, D. B. (2009). Mass media flow and differential distribution of politically disputed beliefs: The belief gap hypothesis. Journalism \& Mass Communication Quarterly, 86(4), 790-808. DOI: https://doi-org.ezproxy.library.wisc.edu/10.1177/107769900908600405.

Hovland, C. I., \& Weiss, W. (1951). The influence of source credibility on communication effectiveness. Public opinion quarterly, 15(4), 635-650.

Jerit, J., \& Barabas, J. (2012). Partisan perceptual bias and the information environment. The Journal of Politics, 74(3), 672-684.

Johnson, T. J., \& Kaye, B. K. (2004). Wag the blog: How reliance on traditional media and the Internet influence credibility perceptions of weblogs among blog users. Journalism \& Mass Communication Quarterly, 81(3), 622-642. DOI: $10.1177 / 107769900408100310$

Johnson, T. J., \& Kaye, B. K. (2014). Credibility of social network sites for political information among politically interested internet users. Journal of Computer-mediated communication, 19(4), 957-974. DOI: $10.1111 /$ jcc4.12084

Kiousis, S. (2001). Public trust or mistrust? Perceptions of media credibility in the information age. Mass Communication \& Society, 4(4), 381-403. DOI: /10.1207/S15327825MCS0404_4

Lazer, D., Baum, M., Grinberg, N., Friedland, L., Joseph, K., Hobbs, W. \& Mattsson, C. ( 2017). Combatting fake news: An agenda for research and action. Shorenstein Center. Retrieved from https://shorensteincenter.org/combating-fake-news-agenda-for-research/. 
Lee, H., Park, S. A., Lee, Y., \& Cameron, G. T. (2010). Assessment of motion media on believability and credibility: An exploratory study. Public Relations Review, 36(3), 310312. doi: 10.1016/j.pubrev.2010.04.003

Lewandowsky, S., Ecker, U. K., \& Cook, J. (2017). Beyond Misinformation: Understanding and Coping with the "Post-Truth" Era. Journal of Applied Research in Memory and Cognition, 6(4), 353-369. DOI: https://doi.org/10.1016/j.jarmac.2017.07.008

Li, X. (2002). Web page design affects news retrieval efficiency. Newspaper Research Journal, 23(1), 38-49.

Lowin, A. (1967). Approach and avoidance: Alternate modes of selective exposure to information. Journal of Personality and Social Psychology, 6(1), 1.

Lowrey, W. (1999). From map to machine: Conceptualizing and designing news on the Internet. Newspaper Research Journal, 20(4), 14-27.

Mackay, J. B., \& Lowrey, W. (2011). The credibility divide: reader trust of online newspapers and blogs. Journal of Media Sociology, 3(1-4), 39-57.

Mahone, J. A. (2014). Attribution has little effect on perceived credibility. Newspaper Research Journal, 35(1), 108-122.

Marsh, E. J., \& Yang, B. W. (2017). A Call to Think Broadly about Information Literacy. Journal of Applied Research in Memory and Cognition, 6(4), 401-404. DOI: https://doi.org/10.1016/j.jarmac.2017.09.012

McDonald, S., \& Stevenson, R. J. (1996). Disorientation in hypertext: the effects of three text structures on navigation performance. Applied ergonomics, 27(1), 61-68. doi: $\underline{10.1016 / 0003-6870(95) 00073-9 .}$. 
Messing, S., \& Westwood, S. J. (2014). Selective exposure in the age of social media: Endorsements trump partisan source affiliation when selecting news online. Communication Research, 41(8), 1042-1063. DOI: 10.1177/0093650212466406

Metzger, M. J. (2007). Making sense of credibility on the Web: Models for evaluating online information and recommendations for future research. Journal of the Association for Information Science and Technology, 58(13), 2078-2091. DOI: 10.1002/asi.20672

Metzger, M. J., Flanagin, A. J., \& Medders, R. B. (2010). Social and heuristic approaches to credibility evaluation online. Journal of communication, 60(3), 413-439. DOI: 10.1111/j.1460-2466.2010.01488.x.

Meyer, P. (1988). Defining and measuring credibility of newspapers: Developing an index. Journalism quarterly, 65(3), 567-574.

Ortutary, B. (2016). "How to spot lies, fake news and propaganda." Associated Press. Retrieved from https://apnews.com/fa3938645328428ea0487290679bca5c.

Pjesivac, I., Geidner, N., \& Cameron, J. (2018). Social credibility online: The role of online comments in assessing news article credibility. Newspaper Research Journal, 39(1), 1831.

Robb, M. (2017). "News and America's kids." Common Sense Media. Retrieved from https://www.commonsensemedia.org/research/news-and-americas-kids.

Sternthal, B., Dholakia, R., \& Leavitt, C. (1978). The persuasive effect of source credibility: Tests of cognitive response. Journal of Consumer research, 4(4), 252-260.

Sundar, S. S. (2008). The MAIN model: A heuristic approach to understanding technology effects on credibility. Digital media, youth, and credibility, 73100. doi: 10.1162/dmal.9780262562324.073. 
Sundar, S. S., Knobloch-Westerwick, S., \& Hastall, M. R. (2007). News cues: Information scent and cognitive heuristics. Journal of the Association for Information Science and Technology, 58(3), 366-378. doi: 10.1002/asi.20511.

Sundar, S. S., \& Nass, C. (2001). Conceptualizing sources in online news. Journal of Communication, 51(1), 52-72. DOI: 10.1111/j.1460-2466.2001.tb02872.x

Tormala, Z. L., Briñol, P., \& Petty, R. E. (2007). Multiple roles for source credibility under high elaboration: It's all in the timing. Social Cognition, 25(4), 536.

Tuchman, G. (1972). Objectivity as strategic ritual: An examination of newsmen's notions of objectivity. American Journal of sociology, 77(4), 660-679

Tully, M., \& Vraga, E. K. (2017). Who Experiences Growth in News Media Literacy and Why Does It Matter? Examining Education, Individual Differences, and Democratic Outcomes. Journalism \& Mass Communication Educator, 1077695817706572.

Vraga, E. K., \& Tully, M. (2015). Media literacy messages and hostile media perceptions: Processing of nonpartisan versus partisan political information. Mass Communication and Society, 18(4), 422-448. 10.1080/15205436.2014.1001910

Vraga, E. K., Edgerly, S., Wang, B. M., \& Shah, D. V. (2011). Who taught me that? Repurposed news, blog structure, and source identification. Journal of Communication, 61(5), 795815.

Westerwick, A., Kleinman, S. B., \& Knobloch-Westerwick, S. (2013). Turn a blind eye if you care: Impacts of attitude consistency, importance, and credibility on seeking of political information and implications for attitudes. Journal of Communication, 63(3), 432-453. DOI: $10.1111 /$ jcom. 12028 
Xu, Q. (2013). Social recommendation, source credibility, and recency: Effects of news cues in a social bookmarking website. Journalism \& Mass Communication Quarterly, 90(4), 757775. DOI: $10.1177 / 1077699013503158$. 